\title{
Red blood cell distribution width may be a new factor that influence the selection of invasive treatment in patients with renal abscess
}

\author{
Mingrui Xia $^{1,2}$, Jun Liu ${ }^{1,2}$, Yang Hong ${ }^{1,2}$, Lizhe $\mathrm{An}^{1,2}$, Liulin Xiong ${ }^{1,2}$, Xiaobo Huang ${ }^{1,2}$, Qingquan Xu ${ }^{1,2}$ \\ ${ }^{1}$ Department of Urology and Lithotripsy Center, Peking University People's Hospital, Beijing, China; ${ }^{2}$ Department of Peking University Applied \\ Lithotripsy Institute, Beijing, China \\ Contributions: (I) Conception and design: Q Xu, M Xia; (II) Administrative support: Q Xu, X Huang; (III) Provision of study materials or patients: \\ J Liu, L Xiong; (IV) Collection and assembly of data: J Liu, M Xia; (V) Data analysis and interpretation: Y Hong, L An, M Xia; (VI) Manuscript \\ writing: All authors; (VII) Final approval of manuscript: All authors. \\ Correspondence to: Qingquan Xu. 133\# Fuchengmen Neidajie Street, XiCheng District, Beijing 100034, China. Email: xuqingquan@bjmu.edu.cn.
}

\begin{abstract}
Backgroundk Red blood cell distribution width (RDW) is associated with the severe morbidity and mortality of some Gram-negative associated chronic diseases. We designed this retrospective study to identify whether RDW can be used as a factor to provide reference for the treatment of renal abscess.

Methods: All patients with renal abscess in our institution between April 2016 and November 2019 were collected, and 38 cases with the characteristic of initially diagnosis and no prior treatment were enrolled. Conservative treatment group and surgical intervention group were set based on the treatment of patients. Univariate analysis and the regression analysis were adopted to identify the difference between these two groups.

Results: Significant differences were found in Diabetes mellitus, RDW and size of renal abscess in both univariate and multifactor analysis. The median value of RDW in all patients was $13.62 \%$, with $13.15 \%$ in conservative treatment group and $14.20 \%$ receiving surgical intervention (OR: 9.48, 95\% CI: 1.15-78.02). Diabetes was present in $42.11 \%$ of patients, with the ratio of $20.0 \%$ in conservative treatment group and $66.67 \%$ in surgical intervention group (OR: 35.22, 95\% CI: 1.55-799.05). The cut-off of RDW and size of renal abscess were $14.07 \%$ and $3.9 \mathrm{~cm}$ respectively.

Conclusions: RDW as an independent predictor of mortality in patients with Gram-negative bacteremia showed differences among patients with renal abscess who received different treatments. Since the relationship between RDW and Gram-negative bacteremia related to mortality, higher initial RDW value may represent a higher severity of renal abscess and a greater likelihood of the need of surgical intervention.
\end{abstract}

Keywords: Renal abscess; red blood cell distribution width (RDW); invasive treatment; Gram-negative bacteremia

Submitted Nov 19, 2020. Accepted for publication Jan 15, 2021.

doi: 10.21037/apm-20-2305

View this article at: http://dx.doi.org/10.21037/apm-20-2305

\section{Introduction}

Renal abscess is a rare urinary system disease confined to the renal parenchyma and characterized by low incidence and high mortality with inappropriate treatment (1-3). The treatment of renal abscess can be divided into simple drug treatment and surgical intervention (4). Simple drug treatment for renal abscess less than $3 \mathrm{~cm}$ and surgical intervention for renal abscess larger than $5 \mathrm{~cm}$ are generally accepted (2,5-9).

There are three main mechanisms of infection including ascending infection, hematogenous spread and direct spread with ascending infection being the most common 
$(5,10)$. Although $S$. aureus was once the most common organism that causing renal abscess, several studies showed that Gram-negative bacteria, represented by E. Coli, had replaced $S$. aureus as the main pathogen $(2,11)$.

Alone with the change of the common causative organisms, some scholars have studied the response of renal abscess to antibiotic therapies to avoid unnecessary invasive treatment. However, waiting for the response may delay early invasive treatment and lead to poor prognosis. Therefore, some indicators related to disease severity in early onset may be a good alternative. Red blood cell distribution width (RDW) is associated with the severe morbidity and mortality of some Gram-negative associated chronic diseases, which has reported by some scholars (12). In view of this point, we designed this retrospective study to identify whether RDW can be used as a factor that to provide reference for the treatment of renal abscess. We present the following article in accordance with the STROBE reporting checklist (available at http://dx.doi. org/10.21037/apm-20-2305).

\section{Methods}

\section{Patients}

All patients with renal abscess in our institution between April 2016 and November 2019 were collected, and 38 cases with the characteristic of initially diagnosis and no prior treatment (antipyretics excepted) were enrolled. Patients without complete image data were excluded. The diagnosis of renal abscess was made by experienced radiologists with the CT performance of abscess confined to the renal cortex, central medulla or calyces (13). Conservative treatment group and surgical intervention group were set based on the treatment of patients. The data of basic characteristics, laboratory examination and imaging data before treatment were collected and the clinical data between two groups were analyzed and compared respectively. The maximum diameter was measured for each abscess as the size of renal abscess with the sum of maximum diameters of all the abscess in one kidney for multiple renal abscesses. Renal dysfunction was defined as estimated glomerular filtration rate less than $90 \mathrm{~mL} / \mathrm{min}^{*} 1.73 \mathrm{~m}^{2}$ and nonfunctional kidney was defined as unilateral estimated glomerular filtration rate less than $10 \mathrm{~mL} / \mathrm{min}^{*} 1.73 \mathrm{~m}^{2}$.

\section{Treatments}

EAU guidelines point out that empirical antibiotic treatment for urological infection was necessary, with fluoroquinolone, group $3 \mathrm{~b}$ cephalosporin or carbapenem being the most common used (14). In our study, both two groups received empirical antibiotic treatment after admission with fluoroquinolones, Group 2, 3 cephalosporin or aminoglycoside. Antibiotics were adjusted on the basis of drug sensitivity test and the response of initial antibiotic therapy. The vast majority of patients got clinically effective treatment, and patients who fail in initial treatment ultimately succeed in further treatment. Clinically effective treatment involved cure and clinical improvement. The standard of cure was the disappearance of clinical symptoms and abnormal examination results. When the clinical symptoms were significantly relieved and laboratory tests got improved, but not yet fully normalized and antibiotic therapy was still required, the outcome was considered as clinical improved (2).

\section{Ethical consideration}

The study was conducted in accordance with the Declaration of Helsinki (as revised in 2013). The study was approved by institutional board of the Peking University People's Hospital (2016-010) and informed consent was taken from all individual participants.

\section{Statistical analyses}

IBM SPSS Statistics Version 25.0 was used for all the data analysis. The independent samples $t$-test, Mann-Whitney $\mathrm{U}$ Test and Fisher's Exact Test were used for different variables. On the basis of univariate analysis, multivariate analysis was carried out for variables with $\mathrm{P}$ value less than 0.2 , and ROC curve was drawn for variables with statistical difference. The standard for statistical difference was $\mathrm{P}$ value less than 0.05 .

\section{Results}

\section{Patient characteristics}

There were 24 (63.16\%) female patients and 14 (36.84\%) male patients. The average age of all the patients with 
Table 1 Patient characteristics of conservative treatment and surgical intervention

\begin{tabular}{|c|c|c|c|c|}
\hline Characteristic & Overall $(n=38)$ & Conservative treatment $(n=20)$ & Surgical intervention $(n=18)$ & $P$ value \\
\hline \multicolumn{5}{|l|}{ Gender, N (\%) } \\
\hline Male & $14(36.84)$ & $7(35.00)$ & $7(38.89)$ & \multirow[t]{2}{*}{0.804} \\
\hline Female & $24(63.16)$ & $13(65.00)$ & $11(61.11)$ & \\
\hline Fever & $29(76.32)$ & $15(75.00)$ & $14(77.78)$ & 1.000 \\
\hline Fever $\geq 38.5^{\circ} \mathrm{C}$ & $8(21.10)$ & $5(25.00)$ & $3(16.70)$ & 0.679 \\
\hline Chills & $6(15.79)$ & $5(25.00)$ & $1(5.56)$ & 0.184 \\
\hline Abdominal pain & $5(13.16)$ & $3(15.00)$ & $2(11.11)$ & 1.000 \\
\hline Nausea and vomiting & $10(26.32)$ & $6(30.00)$ & $4(22.22)$ & 0.719 \\
\hline \multicolumn{5}{|l|}{ Comorbidity, N (\%) } \\
\hline Diabetes & $16(42.11)$ & $4(20.00)$ & $12(66.67)$ & 0.008 \\
\hline Urolithiasis & $16(42.11)$ & $6(30.00)$ & $10(55.56)$ & 0.188 \\
\hline Hepatitis & $6(15.79)$ & $4(20.00)$ & $2(11.11)$ & 0.663 \\
\hline Renal dysfunction & $6(15.79)$ & $1(5.00)$ & $5(27.80)$ & 0.083 \\
\hline
\end{tabular}

renal abscess in this study was $48.87 \pm 15.89$ years old and the distribution was wide, ranging from 22 to 82 years old. The clinical manifestations of renal abscess include fever, lumbago, chills, abdominal pain, nausea, vomiting, with fever $(76.32 \%)$ and lumbago $(52.63 \%)$ being the most common. Diabetes and urolithiasis with the ratio of $42.11 \%$ and $42.11 \%$ respectively were the most common comorbidities in our study. Twenty $(52.63 \%)$ patients received drug therapy only and 18 (47.37\%) patients got surgical intervention. Among all the variables of the basic characteristics, only diabetes showed a significant difference $(\mathrm{P}=0.008)$ between two groups. The data details are represented in Table 1.

\section{Laboratory tests and imaging}

The results of blood and urine test on admission were used as reference data. Variables with $\mathrm{P}$ values less than 0.05 between the two groups included PLT, RDW\%, BUN, PLR, AG and WBC in urine, as shown in Table 2. The median value of initial RDW in all patients was $13.62 \%$, with $13.15 \%$ in conservative treatment group and $14.20 \%$ in invasive treatment group. Significant difference was shown in the size of renal abscess $(2.77 \pm 1.48$ vs. $5.85 \pm$ $2.67 \mathrm{~cm}$ ), with the surgical intervention group being larger $(\mathrm{P}<0.001$, Table 3).

\section{Treatment outcome}

In patients who received conservative treatment, 19 cases showed clinical improvement or cure. While in patients who received surgical intervention initially, 3 patients showed no clinical improvement or cured, with one patient received further treatment of surgical drainage and two patients required nephrectomy after the diagnosis of nonfunctional kidney. Of the 4 patients who failed the initial treatment, 3 had a renal abscess between 3 and $5 \mathrm{~cm}$, and 1 patient with large abscess failed due to long-term use of bone marrow suppressive drugs. After summarizing the characteristics of 4 patients with initial treatment failure, it can be found that 3 patients got the size of renal abscess of $3-5 \mathrm{~cm}$, and 1 patient failed due to long-term use of myelosuppression drugs. 
Table 2 Comparison of laboratory examination in conservative treatment group and surgical intervention group

\begin{tabular}{|c|c|c|c|c|}
\hline Laboratory examination $^{a}$ & Overall $(n=38)$ & Conservative treatment $(n=20)$ & Surgical intervention $(n=18)$ & $P$ value \\
\hline \multicolumn{5}{|l|}{ Blood test } \\
\hline HGB & $108.22 \pm 26.15$ & $115.64 \pm 18.45$ & $99.99 \pm 31.17$ & 0.065 \\
\hline WBC & 8.62 & 8.62 & 8.745 & 0.260 \\
\hline PLT & $285.08 \pm 129.44$ & $236.90 \pm 118.44$ & $338.61 \pm 122.56$ & 0.013 \\
\hline $\mathrm{BUN}, \mathrm{mmol} / \mathrm{L}$ & 4.99 & 4.39 & 5.69 & 0.049 \\
\hline CRE, $\mu \mathrm{mol} / \mathrm{L}$ & 69.5 & 66.5 & 81 & 0.084 \\
\hline NLR & 3.98 & 3.3 & 5.095 & 0.144 \\
\hline PLR & 181.26 & 146.945 & 285.105 & 0.024 \\
\hline$A G$ & $1.11 \pm 0.27$ & $1.21 \pm 0.26$ & $1.01 \pm 0.26$ & 0.021 \\
\hline \multicolumn{5}{|l|}{ Urine test } \\
\hline NIT & $3(7.89)$ & $1(5.0)$ & $2(11.11)$ & 0.584 \\
\hline WBC in urine ${ }^{b}$ & 34.5 & 21 & 77 & 0.022 \\
\hline RBC in urine ${ }^{b}$ & 10 & 8 & 20 & 0.428 \\
\hline
\end{tabular}

a, $\mathrm{N}(\%)$ for the classification variables; mean \pm SD or Median for the continuous variables. ${ }^{\mathrm{b}}$, the WBC in urine was the result per high power lens. HGB, haemoglobin; PLT, platelet count; RDW, distribution width of red blood cells; BUN, blood urea nitrogen; CRE, creatinine; NLR, neutrophil to lymphocyte ratio; PLR, platelet to lymphocyte ratio; TP, total protein; ALB, albumin; AG, albumin to globulin ratio; NIT, nitrite; WBC, white blood cell count; RBC, red blood cell.

Table 3 Comparison of imaging parameters in conservative treatment group and surgical intervention group

\begin{tabular}{lccc}
\hline Imaging parameters & Overall $(\mathrm{n}=38)$ & Conservative treatment $(\mathrm{n}=20)$ & Surgical intervention $(\mathrm{n}=18)$ \\
\hline Location, $\mathrm{N}(\%)$ & & & \\
Left & $22(57.89)$ & $11(55.0)$ & $11(61.11)$ \\
Right & $16(42.11)$ & $9(45.0)$ & $7(38.89)$ \\
Number, N (\%) & & & $11(61.11)$ \\
Single renal abscess & $23(60.53)$ & $12(60.0)$ & $7(38.89)$ \\
Multiple renal abscess & $15(39.47)$ & $8(40.0)$ & 5.000 \\
Size, cm, mean \pm SD & $4.23 \pm 0.27$ & $2.77 \pm 1.48$ & $7(38.89)$ \\
Perirenal abscess ${ }^{\mathrm{a}}, \mathrm{N}(\%)$ & $13(34.21)$ & $6(30.0)$ & $<0.001$ \\
\hline
\end{tabular}

${ }^{\mathrm{a}}$, renal abscess combined with perirenal abscess.

\section{Urine and abscess culture}

Urine culture was performed in 21 patients, of which 14 $(66.67 \%)$ were negative and $7(33.33 \%)$ were positive. Five of the positive patients (71.43\%) were Gram-negative bacteria. Abscess culture was performed in 15 patients $(83.33 \%)$ who received surgical intervention, of which 12 (80.00\%) were positive, with Gram-negative bacteria being found in $11(91.67 \%)$ cases. 


\section{Logistic regression analysis and $R O C$ curve}

On the basis of univariate analysis, logistic regression analysis was carried out for variables with $\mathrm{P}$ value less than 0.2. Diabetes mellitus (OR: 35.22, 95\% CI: 1.55-799.05), size of renal abscess (OR: 2.67, 95\% CI: 1.00-7.11) and RDW (OR: 9.48, 95\% CI: 1.15-78.02) were retained in the regression equation, with $\mathrm{P}$ value less than 0.05 . It was presented in Table 4. ROC curves were drawn for variables of RDW and size (Figure 1). The cut-off of RDW was $14.07 \%$, with sensitivity 90.00 and specificity 55.56. In addition, the cut-off of size of renal abscess was $3.9 \mathrm{~cm}$, with sensitivity 95.00 and specificity 77.78 .

\section{Discussion}

Renal abscess characterized by low incidence and high mortality is a clinically important diagnosis during clinical work. The update of examination technique (CT, MRI) has improved the accuracy of the diagnosis of renal abscess and the prognosis of renal abscess is related to the early

Table 4 Results of logistic regression analysis with respect to probability of surgical intervention

\begin{tabular}{lccc}
\hline Variable & OR & $95 \% \mathrm{Cl}$ & $\mathrm{P}$ value \\
\hline Diabetes mellitus & 35.22 & $1.55-799.05$ & 0.025 \\
RDW & 9.48 & $1.15-78.02$ & 0.037 \\
Size & 2.67 & $1.00-7.11$ & 0.049 \\
\hline
\end{tabular}

OR, odds ratio; $\mathrm{Cl}$, confidence interval; RDW, red blood cell distribution width. and accurate diagnosis (15). In 1996, Siegel proposed for the first time that 3 and $5 \mathrm{~cm}$ can be used as a reference standard for surgical intervention of renal abscess (9). After his proposal, many researchers had further studied the relationship between the abscess size and surgical intervention. However, no definite consensus of the proper treatment has been reached, especially for the size of $3 \sim 5 \mathrm{~cm}(3,6-8,16)$. Nevertheless, consensus on the use of antibiotics for renal abscess, an infectious disease, has been unanimously reached.

Recently, Gram-negative bacteria gradually replaced Staphylococcus aureus and became the main pathogenic bacteria of renal abscess due to the use of antibiotic $(2,11)$. In a study conducted by Yamamichi et al., E. coli was responsible for $23 \%$ of renal abscesses (17). Although there were some negative results, our study also found that Gram-negative bacteria was the main causative organism. In the meanwhile, some scholars found that the increase of RDW to some extent indicates the severity and mortality of Gram-negative bacteria diseases (12). In view of this point, we speculated whether initial RDW can be used to indicate the severity of renal abscess and guide surgical intervention. In our study, the median value of initial RDW in all patients was $13.62 \%$, with $13.15 \%$ in conservative treatment group and $14.20 \%$ in invasive treatment group. Compared with the conservative group, patients receiving surgical intervention appeared to have higher initial RDW values. After excluding other factors through multivariate analysis, it was found that RDW was still the influential factor that may influence the choice of surgical intervention for renal abscess. This may be attributed to the changes

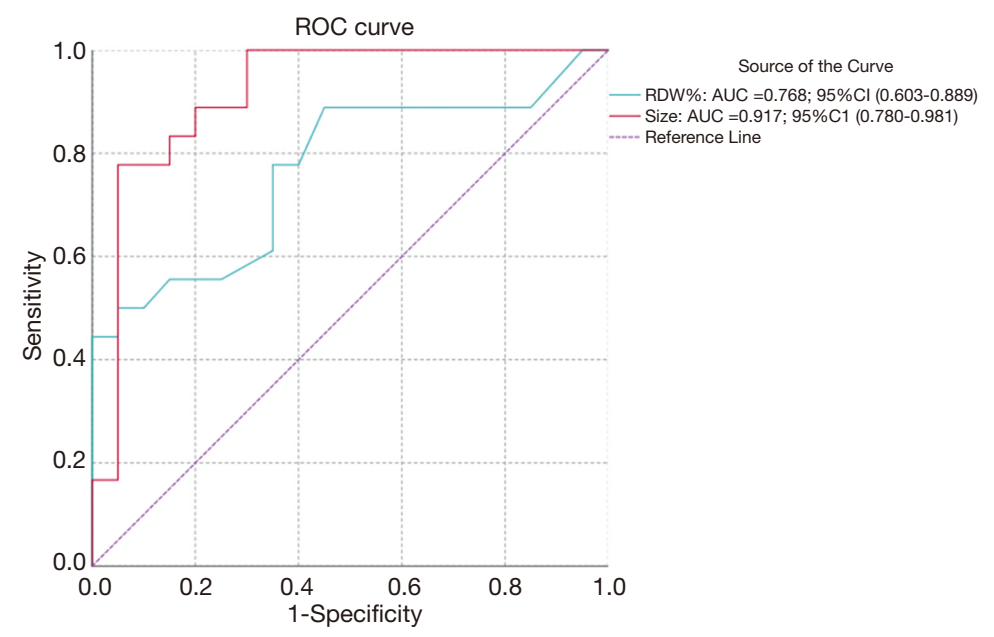

Figure 1 ROC curve of RDW and size of renal abscess. RDW, red blood cell distribution width. 
of the predominant pathogens of renal abscess. To our knowledge, for the first time, the increase of initial RDW has been considered as an influential factor that guiding the treatment of renal abscess.

Although the patients of renal abscess receiving surgical intervention with higher initial RDW was found for the first time in our study, its concrete pathophysiologic mechanism is still unknown, the role of RDW in predicting severity and mortality in patients with Gram-negative bacteremia may be one explanation. It has been generally established that, Gram-negative bacteria can cause systemic inflammation which can influence bone marrow function and iron metabolism $(18,19)$, thus lead to the suppression of erythrocyte maturation. When inflammation is severe, even sepsis, the newer and larger reticulocytes are allowed to enter the circulation, consequently cause the increase of RDW $(20,21)$, which may explain why RDW was higher in patients with renal abscess who needed invasive treatment. Oxidative stress may also one reason for the elevated RDW $(22,23)$. To sum up, higher initial RDW value may represent a higher severity of renal abscess and a greater likelihood of the need of invasive treatment.

In additional to RDW, the difference was also found in diabetes mellitus ( $\mathrm{P}$ value 0.008 ). In our study, diabetes mellitus occurred in $42.11 \%$ of patients, with the ratio of $20.0 \%$ in conservative treatment group and $66.67 \%$ in surgical intervention group. Diabetes can increase the risk of infection and control difficulty (24). This may be one of the reasons why renal abscess patients with diabetes received more surgical intervention. Research by Ko et al. may also explain this phenomenon (25).

Some studies also reported that the poor prognosis and high mortality of renal abscess are related to some other factors such as advanced age, higher C-reactive protein levels and blood urea nitrogen, occurrence of lethargy $(6,26)$. In our study, the mean age was $48.87 \pm 15.89$ years, the ratio of female was $63.16 \%$. Although the youngest renal abscess patient was reported to be only 6 months old (27), no pediatric patient was included. In our study, the average value of PLT was $285.08 \pm 129.44$, with patients receiving invasive treatment had higher value which was contrary to the outcome of poor prognosis indicated by thrombocytopenia (3). This may be related to the bias of subjective choice in clinical work.

In addition to the above variables, BUN, PLR, AG and $\mathrm{WBC}$ in urine with differences in univariate analysis were excluded in multivariate analysis. Diabetes mellitus, size of renal abscess and RDW were retained in the regression equation and ROC curves were drawn for variables of RDW and size. In general, for patients with RDW $>14.07 \%$, abscess size $>3.9 \mathrm{~cm}$ and diabetes mellitus, surgical intervention should be taken into consideration.

However, there were several limitations to our study. Firstly, there is a certain degree of subjective bias in this retrospective study, especially in the choice of initial treatment. Secondly, the number of cases is limited due to single center study. Since renal abscess is a rare urinary system disease, more cases and multicenter study are needed. Thirdly, some factors such as C-reactive protein, erythrocyte sedimentation rate which were demonstrated to be associated with prognosis were excluded due to incomplete data. Lastly, blood culture was not been discussed for the reason of limited data.

\section{Conclusions}

With Gram-negative bacteremia being the most common pathogenic bacteria of renal abscess, RDW as an independent predictor of mortality in patients with Gramnegative bacteremia showed differences among patients with renal abscess who had received different treatments. Higher initial RDW value may represent a higher severity of renal abscess and a greater likelihood of the need of invasive treatment.

\section{Acknowledgments}

Funding: None.

\section{Footnote}

Reporting Checklist: The authors have completed the STROBE reporting checklist. Available at http://dx.doi. org/10.21037/apm-20-2305

Data Sharing Statement: Available at http://dx.doi. org/10.21037/apm-20-2305

Conflicts of Interest: All authors have completed the ICMJE uniform disclosure form (available at http://dx.doi. org/10.21037/apm-20-2305). The authors have no conflicts of interest to declare. 
Ethical Statement: The authors are accountable for all aspects of the work in ensuring that questions related to the accuracy or integrity of any part of the work are appropriately investigated and resolved. The study was conducted in accordance with the Declaration of Helsinki (as revised in 2013). The study was approved by institutional board of the Peking University People's Hospital (2016010) and informed consent was taken from all individual participants.

Open Access Statement: This is an Open Access article distributed in accordance with the Creative Commons Attribution-NonCommercial-NoDerivs 4.0 International License (CC BY-NC-ND 4.0), which permits the noncommercial replication and distribution of the article with the strict proviso that no changes or edits are made and the original work is properly cited (including links to both the formal publication through the relevant DOI and the license). See: https://creativecommons.org/licenses/by-nc-nd/4.0/.

\section{References}

1. Gardiner RA, Gwynne RA, Roberts SA. Perinephric abscess. BJU Int 2011;107 Suppl 3:20-3.

2. Coelho RF, Schneider-Monteiro ED, Mesquita JL, et al. Renal and perinephric abscesses: analysis of 65 consecutive cases. World J Surg 2007;31:431-6.

3. Lin HS, Ye JJ, Huang TY, et al. Characteristics and factors influencing treatment outcome of renal and perinephric abscess--a 5-year experience at a tertiary teaching hospital in Taiwan. J Microbiol Immunol Infect 2008;41:342-50.

4. Shu T, Green JM, Orihuela E. Renal and perirenal abscesses in patients with otherwise anatomically normal urinary tracts. J Urol 2004;172:148-50.

5. Lee SH, Jung HJ, Mah SY, et al. Renal abscesses measuring $5 \mathrm{~cm}$ or less: outcome of medical treatment without therapeutic drainage. Yonsei Med J 2010;51:569-73.

6. Hung CH, Liou JD, Yan MY, et al. Immediate percutaneous drainage compared with surgical drainage of renal abscess. Int Urol Nephrol 2007;39:51-5.

7. Dalla Palma L, Pozzi-Mucelli F, Ene V. Medical treatment of renal and perirenal abscesses: CT evaluation. Clin Radiol 1999;54:792-7.

8. Lee BE, Seol HY, Kim TK, et al. Recent clinical overview of renal and perirenal abscesses in 56 consecutive cases.
Korean J Intern Med 2008;23:140-8.

9. Siegel JF, Smith A, Moldwin R. Minimally invasive treatment of renal abscess. J Urol 1996;155:52-5.

10. Constantine S, Kaye J. Metastatic renal abscess mimicking Wilms' tumour. Pediatr Radiol 2004;34:924-6.

11. Capitán Manjón C, Tejido Sánchez A, Piedra Lara JD, et al. Retroperitoneal abscesses--analysis of a series of 66 cases. Scand J Urol Nephrol 2003;37:139-44.

12. Ku NS, Kim HW, Oh HJ, et al. Red blood cell distribution width is an independent predictor of mortality in patients with gram-negative bacteremia. Shock 2012;38:123-7.

13. Patterson JE, Andriole VT. Renal and perirenal abscesses. Infect Dis Clin North Am 1987;1:907-26.

14. 3D.4 Disease Management; in Guidelines on Urological Infections. European Association of Urology. 2015:2425. Available online: https://uroweb.org/guidelines/

15. Rubilotta E, Balzarro M, Lacola V, et al. Current clinical management of renal and perinephric abscesses: a literature review. Urologia 2014;81:144-7.

16. Linder BJ, Granberg CF. Pediatric renal abscesses: A contemporary series. J Pediatr Urol 2016;12:99.e1-5.

17. Yamamichi F, Shigemura K, Kitagawa K, et al. Should We Change the Initial Treatment of Renal or Retroperitoneal Abscess in High Risk Patients? Urol Int 2017;98:222-7.

18. Deswal A, Petersen NJ, Feldman AM, et al. Cytokines and cytokine receptors in advanced heart failure: an analysis of the cytokine database from the Vesnarinone trial (VEST). Circulation 2001;103:2055-9.

19. Chiari MM, Bagnoli R, De Luca PD, et al. Influence of acute inflammation on iron and nutritional status indexes in older inpatients. J Am Geriatr Soc 1995;43:767-71.

20. Rogiers P, Zhang H, Leeman M, et al. Erythropoietin response is blunted in critically ill patients. Intensive Care Med 1997;23:159-62.

21. Pierce CN, Larson DF. Inflammatory cytokine inhibition of erythropoiesis in patients implanted with a mechanical circulatory assist device. Perfusion 2005;20:83-90.

22. Kolls JK. Oxidative stress in sepsis: a redox redux. J Clin Invest 2006;116:860-3.

23. Ghaffari S. Oxidative stress in the regulation of normal and neoplastic hematopoiesis. Antioxid Redox Signal 2008;10:1923-40.

24. Stapleton A. Urinary tract infections in patients with diabetes. Am J Med 2002;113 Suppl 1A:80S-84S.

25. Ko MC, Liu CC, Liu CK, et al. Incidence of renal and perinephric abscess in diabetic patients: a population-based 
national study. Epidemiol Infect 2011;139:229-35.

26. Yen DH, Hu SC, Tsai J, et al. Renal abscess: early diagnosis and treatment. Am J Emerg Med 1999;17:192-7.

Cite this article as: Xia M, Liu J, Hong Y, An L, Xiong L, Huang X, Xu Q. Red blood cell distribution width may be a new factor that influence the selection of invasive treatment in patients with renal abscess. Ann Palliat Med 2021;10(4):3916-3923. doi: 10.21037/apm-20-2305
27. Wang YT, Lin KY, Chen MJ, et al. Renal abscess in children: a clinical retrospective study. Acta Paediatr Taiwan 2003;44:197-201. 